\title{
CARACTERIZAÇÃO DAS PROPRIEDADES TÉRMICAS DE FIBRA DE RAMI POR TÉCNICAS FOTOTÉRMICAS
}

\author{
Alice Barreto Bevitori \\ Mestre em Engenharia e Ciência dos Materiais / UENF \\ alicebevitori@gmail.com \\ Isabela Leão Amaral da Silva \\ Mestre em Engenharia e Ciência dos Materiais / UENF \\ isabelaleao@gmail.com
}

Recebido: 04 de dezembro de 2011. Revisado: 27 de setembro de 2012. Aceito: 22 de janeiro de 2013. Publicado online: 23 de março de 2013.

\section{RESUMO}

As fibras naturais estão atraindo interesse nos setores de engenharia, devido às vantagens específicas, tais como baixa densidade e custo, assim como a vantagem de ser renovável, biodegradável neutra em relação à emissão de $\mathrm{CO}_{2}$ e poder ser reciclada.. Em particular, a fibra extraída do caule da planta de rami é reconhecida atualmente como uma das mais fortes fibras lignocelulósicas. Entretanto, as propriedades térmicas da fibra de rami ainda não foram plenamente investigadas. No presente trabalho, teve-se por objetivo investigar por técnicas de espectroscopia fotoacústica e fototérmicas as propriedades de difusividade, capacidade térmica específica e condutividade térmica de amostras de fibras de rami prensadas. Os resultados revelaram que estas fibras apresentam capacidade superior de isolamento térmico.

Palavras-chave: Fibra de rami; Difusividade térmica; Calor específico; Condutividade térmica.

\begin{abstract}
Natural fibers are attracting the interest of engineering sectors owing to specific advantages such as lightweight and lower cost as well as for their inherent condition of being renewable, biodegradable, recyclable and neutral with respect to $\mathrm{CO}_{2}$ emission. In particular, the fiber extracted from the bast of the ramie plant is nowadays recognized as one of the strongest lignocellulosic fibers. However, the thermal properties of the ramie fibers have yet not been fully investigated. The present work had as its objective to investigate, by photoacoustic spectroscopy and photothermic techniques the thermal properties of diffusivity, specific capacity and conductivity of pressed ramie fibers samples. The results revealed that these fibers showed a superior thermal insulation capacity.
\end{abstract}

Keywords: Ramie fibers; Thermal diffusivity; Specific heat; Thermal conductivity. 


\section{INTRODUÇÃO}

As aplicações de fibras naturais lignocelulósicas à base de celulose de plantas estão recebendo uma elevada atenção como alternativa mais correta para substituir materiais não-recicláveis e de consumo intensivo de energia como a fibra de vidro, para reforço de compósitos ${ }^{(1-4)}$. A utilização de fibras naturais apresenta ainda outras vantagens em relação à fibra de vidro, como por exemplo, o custo mais barato e devido às fibras naturais serem mais leves e não abrasivas aos equipamentos de processamento. Vale ressaltar ainda que a fibra de vidro representa um risco para a saúde quando inalada e sua produção está associada com emissões de $\mathrm{CO}_{2}$. Nenhuma destas deficiências se aplica às fibras lignocelulosicas extraídas de plantas, que são renováveis, biodegradáveis e neutras relativamente à emissão de $\mathrm{CO}_{2}$, que se acredita ser a principal causa do efeito estufa na terra e, por extensão, responsável pelo aquecimento global ${ }^{(5)}$.

A fibra natural lignocelulósica extraida do caule da planta de rami (Boehmeria nivea), ilustrada na Fig. 1, pode atingir altos valores de resistência à tração ${ }^{(6)}$, além de não causar problemas ao meio ambiente.
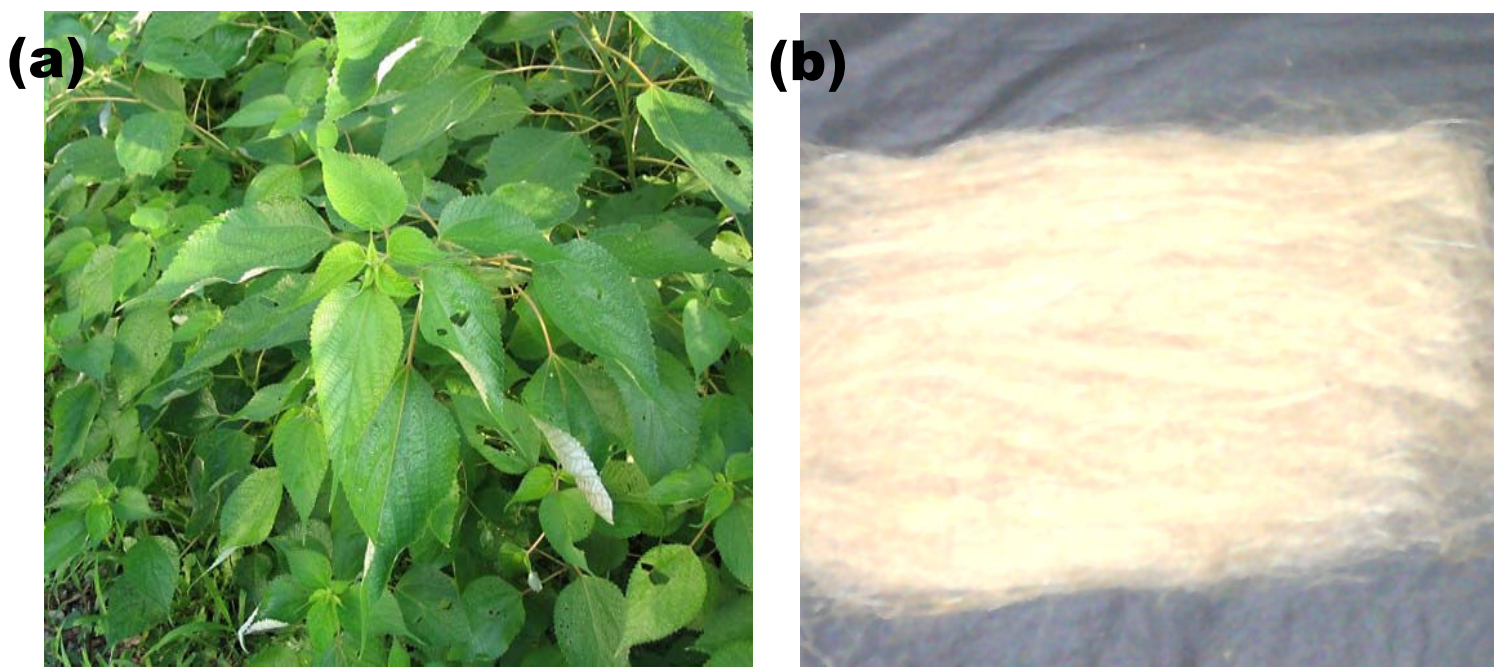

Figura 1. Planta de rami (a) e as fibras de rami (b).

Uma investigação preliminar sobre o comportamento térmico da fibra de rami por análise termogravimétrica, TGA, e seus derivados, DTG, bem como calorimetria exploratória diferencial, DSC, revelou as condições paramétricas associadas com a perda de água, decomposição da celulose e lignina, e também reações de oxidação de elementos como cálcio e silício ${ }^{(7)}$.

A espectroscopia fotoacústica e técnicas fototérmica são técnicas já estabelecidas para caracterizar propriedades térmicas de materiais isolantes. Estas técnicas são baseadas na geração de calor como resultado da absorção de uma radiação ${ }^{(8,9)}$, e foram aplicados aos materiais poliméricos ${ }^{(8)}$. Assim, o objetivo do presente trabalho foi realizar uma caracterização preliminar fotoacústica e fototérmica da fibra de rami.

\section{MATERIAL E MÉTODOS}

As fibras de rami utilizadas neste trabalho foram adquiridas pela empresa brasileira Sisalsul. Fibras como as mostradas na Fig. 1 foram analisadas estatisticamente seguindo o procedimento descrito em outro trabalho ${ }^{(10)}$. Em suma, as fibras possuem uma distribuição de tamanho de 20 - $200 \mu \mathrm{m}$ com um valor médio de $97 \mu \mathrm{m}$. 
A preparação da amostra fotoacústica está associada a um processo cuidadoso, no qual as fibras de rami são moídas e prensadas na forma de discos com espessura menor que $0.5 \mathrm{~mm}$, como observada na Fig. 2 (a). Cada amostra foi testada em uma célula fotoacústica aberta, como mostrada na Fig. 2 (b), o método de elevação fototérmica sob iluminação de laser contínuo também foi empregado. Ambas as técnicas permitiram determinar a difusividade térmica, $\boldsymbol{\alpha}_{\mathrm{s}}$, e a capacidade térmica específica $\boldsymbol{\rho} \mathbf{c}_{\mathrm{p}}$. A partir desses parâmetros, a condutividade térmica, $\mathrm{k}$, da fibra de rami foi avaliada pela seguinte equação

$$
k=\alpha_{s} \rho c_{p}
$$

A difusividade térmica foi medida pela técnica fotoacústica, que considera a perda de calor em uma amostra devido a um processo de relaxamento térmico após a absorção da luz. A célula de fotoacústica aberta ("open photoacustic cell" - OPC) consiste em montar uma amostra, Fig. 2 (b), diretamente em um microfone cilíndrico. A câmara de ar da frente do microfone funciona como câmara de gás convencional, normalmente aplicada em outras técnicas fotoacústica ${ }^{(9)}$. A interpretação dos resultados é baseada no fato de que o método OPC foi o mecanismo termoelástico dominante. $\mathrm{O}$ valor da $\alpha_{\mathrm{s}}$ foi então obtido a partir da montagem de dados do procedimento experimental, $\phi$, através das seguintes equações

$$
\begin{aligned}
& \phi=\phi_{\mathrm{o}}+\arctan \left(\frac{1}{\mathrm{x}-1}\right) \\
& \mathrm{x}=\ell_{\mathrm{s}}\left(\frac{\pi \mathrm{f}}{\alpha_{\mathrm{s}}}\right)^{1 / 2}
\end{aligned}
$$

onde $\ell_{\mathrm{s}}$ é a espessura da amostra e f a frequência do sinal fotoacústico. Supõe-se que a amostra é oticamente opaca e que o fluxo de calor no ar circundante é desprezível.

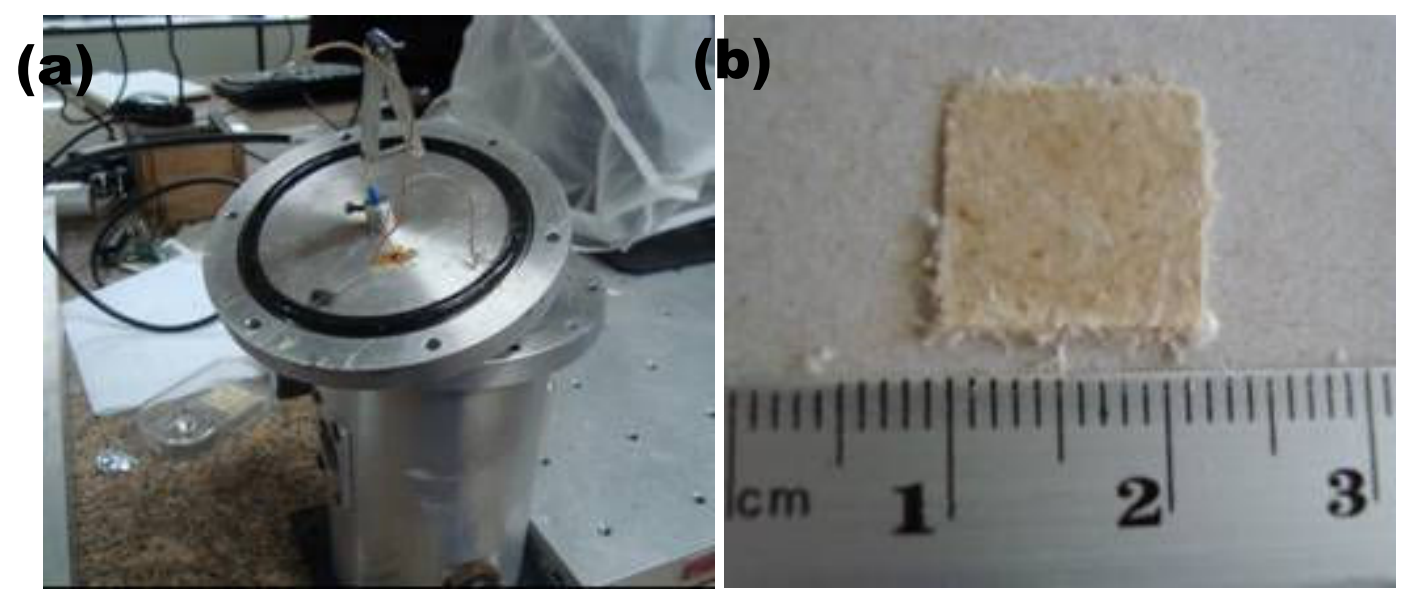

Figura 2. Aparelho de célula aberta (a) para a medição térmica e amostra fotoacústica (b). 


\section{RESULTADOS E DISCUSSÕES}

A Fig. 3 apresenta uma das curvas típicas de variação da fase $\phi$ do sinal fotoacústico com sua frequência $\mathrm{f}$. Vale aqui mencionar que a fase do sinal é a diferença de fase entre o sinal gerado e o detectado. Por sua vez, a frequência colocada como variável independente na Fig. 3 é a frequência de modulação do laser.

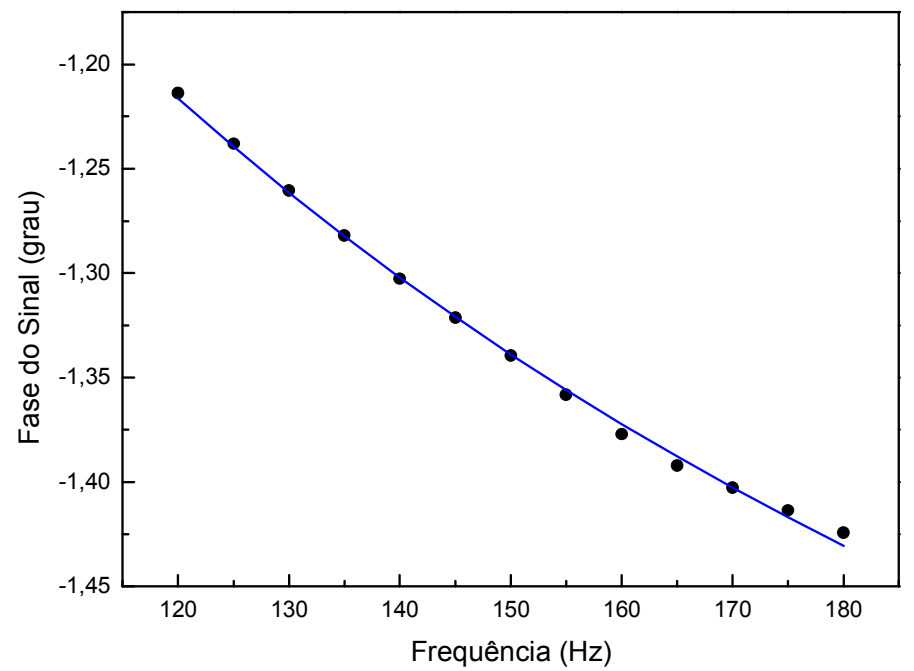

Figura 3. Variação da fase $\phi$ do sinal fotoacústico com a frequência $\mathrm{f}$ para a fibra de rami.

$\mathrm{Na}$ Fig. 3, os pontos pretos representam os dados experimentais enquanto a linha contínua corresponde à curva de melhor ajuste para a trajetória destes pontos experimentais. Considerando-se que a espessura de cada amostra é de $\ell_{\mathrm{s}}=0.5 \mathrm{~mm}$, a difusividade térmica, baseada na Eq (3), foi determinada como o seguinte valor médio

$$
\alpha_{\sigma}=\cong(0,4 \pm 0,1) \mathrm{cm}^{2} \cdot \mathrm{s}^{-1}
$$

Tendo em vista a evolução com o tempo da amostra de rami quando a iluminação com laser é apagada, construíram-se curvas experimentais como a exemplificada na Fig. 4. Nesta figura, os pontos indicados por círculos abertos correspondem aos dados experimentalmente obtidos. Por outro lado, a linha contínua representa a curva de melhor ajuste matemático para a trajetória dos pontos experimentais. Esta linha contínua foi calculada considerando-se as seguintes equações ${ }^{(10,11)}$

$$
\Delta T=\left(\frac{I_{0} \tau}{\ell_{s} \rho c_{p}}\right)\left(1-e^{-t / \tau}\right)
$$

onde $\mathrm{I}_{\mathrm{o}}$ é a intensidade do laser incidente sobre a amostra e 


$$
\tau=\ell_{\mathrm{s}} \rho \mathrm{c}_{\mathrm{p}}(2 \mathrm{H})
$$

é conhecido como o tempo de aumento da temperatura ("rise temperature time"), no qual

$$
\mathrm{H}=4 \sigma \mathrm{T}_{\mathrm{o}}{ }^{3}
$$

é denominado o coeficiente de transferência de calor irradiado, e se compõem da constante de StefanBoltzmann, $\sigma$, bem como da temperatura ambiente $T_{0}$. Utilizando-se o valor de $\tau$, como um parâmetro ajustável na curva da Fig. 4, a capacidade térmica específica pode ser obtida.

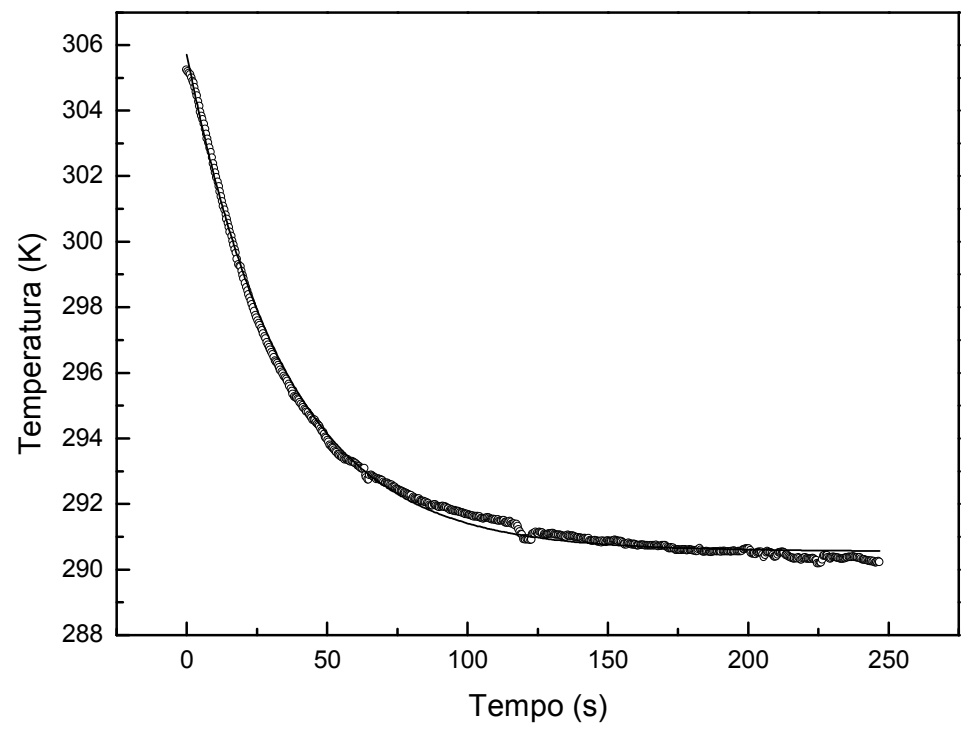

Figura 4. Evolução da temperatura com o tempo na parte de trás da superfície da amostra após o desligamento da iluminação.

Assim, considerando as Eq 5, 6 e 7, o valor médio da capacidade térmica específica da fibra de rami foi obtida como:

$$
\rho_{\mathrm{c}}=(0,7 \pm 0,1) \mathrm{J} \cdot \mathrm{K}^{-1} \cdot \mathrm{cm}^{-3}
$$

Para completar esta caracterização térmica, obteve-se a condutividade térmica da fibra de rami utilizando-se os valores da difusividade térmica, Eq (4) e da capacidade térmica específica, Eq (8) conjuntamente na Eq (1) 


$$
\mathrm{k}=(0,3 \pm 0,1) \mathrm{W} \cdot \mathrm{cm}^{-1} \cdot \mathrm{K}
$$

Este valor é comparável a valores obtidos para os materiais poliméricos ${ }^{(12)}$. Embora os resultados obtidos no presente trabalho sejam preliminares e levem em consideração somente a média de três réplicas, o valor da condutividade térmica na Eq (9) indica que a fibra de rami tem características de um bom isolante térmico.

\section{CONCLUSÃO}

Propriedades fototérmicas da fibra de rami extraídas do caule da planta (Boehmenia nívea) foram caracterizadas por espectroscopia fotoacústica.

A difusividade térmica $\alpha_{\mathrm{s}}=(0,4 \pm 0,1) \mathrm{cm}^{2} . \mathrm{s}^{-1}$ e a capacidade térmica específica $\rho_{\mathrm{c}}=(0,7 \pm 0,1) \mathrm{J} . \mathrm{K}^{-}$ ${ }^{1} . \mathrm{cm}^{-3}$ foram obtidas com razoável precisão a partir de ajustes matemáticos de dados experimentais.

Finalmente, calculou-se a condutividade térmica $\mathrm{k}=(0,3 \pm 0,1) \mathrm{W} \cdot \mathrm{cm}^{-1} \cdot \mathrm{K}$, que indica ser a fibra de rami um bom isolante térmico.

\section{AGRADECIMENTOS}

Os autores agradecem ao suporte financeiro e técnico da UENF, CAPES e FAPERJ, bem como bolsas fornecidas por estas instituições.

\section{REFERÊNCIAS}

MONTEIRO, S.N., LOPES, F.P.D., FERREIRA, A.S., NASCIMENTO, D.C.O. Natural fiber polymer matrix composites: cheaper, tougher and environmentally friendly, JOM, 61 (2009) 17-22.

WAMBUA, P., IVENS, I., VERPOEST, I. "Natural fibers: can they replace glass and fiber reinforced plastics?”, Composites Science and Technology, 63 (2003) 1259 - 1264.

CROCKER, J., "Natural materials innovative natural composites". Materials Technology, 2-3 (2008) 174178.

MOHANTY, A.K., MISRA, M., HINRICHSEN, G., "Biofibers, biodegradable polymers and biocomposites: an overview", Macromolecular Mat. And Engineering, 276/277 (2000), 1-24.

GORE, A., "An Inconvenient Truth. The Planetary Emergency of Global Warming and What We Can do About It”. Emmaus, Pennsylvania, USA: Rodale Press, 2006.

MONTEIRO, S. N., SATYANARAYANA, K. G., LOPES, F. P. D., High strength natural fibers for improved polymer matrix composites, Materials Science Forum, 638-642 (2010) 961-966.

Persp. online: exatas \& eng., Campos dos Goytacazes, 3 (5), 15 - 21 , 2013 
BEVITORI, A.B., RODRIGUEZ, R.S., SILVA, I.L.A., LOPES, F.P.D., MONTEIRO, S.N., Thermal Analysis characterization of ramie fibers with different diameters. PROCEEDINGS OF THE TMS CONFERENCE, (San Diego, CA, USA, March, 2011) (2011) 1-6.

ALMOND, D., PATEL P., "Photoacoustic and Photothermal Science and Techniques". Chapman and Hall: London, 1996.

VARGAS, H., MIRANDA L. C. M., Photoacoustic and related photothermal techniques. PHYS. REP. 161(2) (1988) 43-101.

MARGEM, F. M., MONTEIRO, S. N., NETO, J. B., RODRIGUEZ, R. J. S., SOARES, B. G., The dynamic mechanical behavior of epoxy matriz composites reinforced with ramie fibers, REV. MATER. 15 (2) (2010) 167-175.

VARGAS, H., MIRANDA, L. C. M., Photoacoustic and related photothermal techniques. PHYS. REP. 161(2) (1988) 43-101.

CALLISTER, W. D., Ciência e Engenharia de Materiais: Uma Introdução. 5ed. LTC, São Paulo (2002). 